\title{
Numerical Simulation of VHF Radar Echoes of Sea Ice Based on Mueller Matrix Solution
}

\author{
Zhao Yao' \\ ${ }^{1}$ Department of Communication Science and Engineering, Fudan University, Shanghai 200433, China
}

\begin{abstract}
Based on the time domain Mueller matrix solution, a method for VHF (very high frequency, 30-300MHz) radar pulse penetration on sea ice, programming by Visual $\mathrm{CH}$, is developed. The polarimetric radar pulse echoes contain echoes from top and bottom interfaces, air-bubble's volumetric scattering and their multiinteractions. By changing model parameters including the layer thickness, salinity, dielectric constant and interfacial roughness, the radar pulse echoes on sea ice are numerically simulated to verify the feasibility of this method. The results demonstrate that the model can be used to explore sea ice, of which rich information such as depth and other structure properties can be revealed.

Index Terms - Scattering model; Mueller matrix solution; Sea ice layer exploration; Numerical simulation.
\end{abstract}

\section{Introduction}

Sea ice includes salt water ice (by sea water freezing), continental glacier, river ice and lake ice. In the field of microwave remote sensing, according to the stage of development, sea ice can be classified as new-born ice, slud, one-year ice and multi-year ice; according to the motion state, sea ice can be classified as land ice and floating ice. Land ice (smooth surface) generally froze with coast and extend outwards, the margin of which can move with tidal waves. Floating ice is always the primary ice, floating in the sea, moving with wind or ocean current. As an important part of the marine resources development, sea ice has a great impact on global climate and coastal engineering construction. Since 1940s, high latitude countries started the sea ice observation and research work. By observations through shore station, vehicle and aircraft and measurements by sonar, radar and satellite, methods of sea ice and iceberg detection by mathematical statistics and dynamic numerical methods are developed for sea ice forecast and warning of danger are released.

In recent decades, spaceborne and airborne remote sensing, along with other earth observing technologies, are applied in atmospheric science, oceanography, disaster monitoring and other research fields [2]. Traditional remote sensing observation of sea ice usually use visual and infrared remote sensing image, such as NOAA, AVHRR, MODIS and $\mathrm{SSM} / \mathrm{I}$ data. In recent years, microwave remote sensing in allweather and day-and-night observation is developing rapidly. For example, the Synthetic Aperture Radar (SAR), such as ESA Environment Satellite ENVISAT ASAR (C band), ALOS land observation satellite in Japan with PALSAR (L band), has the advantages of high resolution and capacity of all-weather and day-and-night observation. However, the studies above focus on the detection of changes in sea ice image, which lack information of sea ice layer and beneath information. The VHF radar, which is not sensitive to clouds and sun light, can provide high resolution remote sensing images in all weather. The VHF radar is sensitive to roughness and salinity of sea ice, which is valid to identify different types and classify characteristics. Therefore, in order to monitor sea ice change dynamically and provide disaster warnings, we must use Very High Frequency (VHF, 100-300 MHz ) radar.

\section{Sea ice simulation model and theory}

This paper proposes a method for VHF radar to detect the structure of sea ice layer. Sea ice is a mixture of pure ice, nonspherical salt solution impurities and air bubbles with random distribution. Dielectric properties of salt solution impurities and air bubbles affect the dielectric properties of sea ice greatly [3], and especially increase the scattering properties of sea ice. In this paper, the sea ice is regarded as a lossy dielectric layer, which has random rough surface and bottom. Mixture of pure ice and salt solution impurity is the background medium, while air bubbles with random distribution and orientation in the sea ice layer are scatterers. We constructed the Mueller matrix solution of the model from vector radiative transfer (VRT) equation. By changing the dielectric parameters of sea ice, we obtain the numerical simulation radar echoes. VHF radar can detect differences of radar echoes under different conditions of sea ice, while the layer structure and physical characteristics can be revealed.

As shown in Fig.1, the sea ice layer (1) is a lossy medium of pure ice and salt solution impurity with thickness d, and air bubbles scatterers overlays the bottom of sea ice (2).

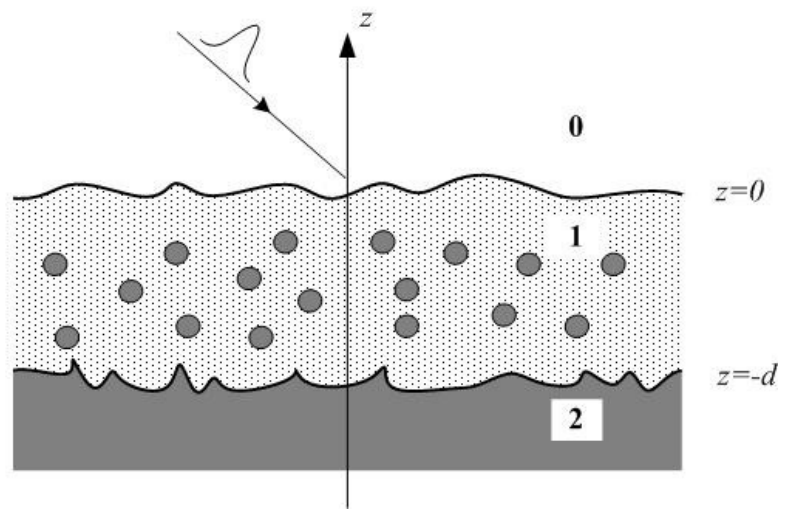

Fig. 1 Geometry of sea ice model 
In general, the sea surface under sea ice layer is a random rough surface, where surface scattering plays a major role. This paper cites the method of numerical simulation of scattering from a layer of hybrid particles above a randomly rough surface, proposed in reference [4]. The VRT equation iscited from [5].Because the underlying surface is rough surface, the boundary conditions should be changed to:

$$
\begin{aligned}
& \mathbf{I}(\theta, \phi, z=-d)=\int_{0}^{2 \pi} d \phi^{\prime \prime} \int_{0}^{\pi / 2} d \theta^{\prime \prime} \sin \theta^{\prime \prime} \overline{\mathbf{M}}_{f}\left(\theta, \phi ; \theta^{\prime \prime}, \phi^{\prime \prime}\right) \\
& \mathbf{I}\left(\theta^{\prime \prime}, \phi^{\prime \prime}, z=-d\right)
\end{aligned}
$$

$\delta$ is the impulse function, $\overline{\mathbf{M}}_{f}$ is mueller matrix for rough surface scattering, f means rough surface.

The Mueller matrix including surface scattering and volume scattering:

$$
\begin{aligned}
& \overline{\mathbf{M}}\left(\Theta, \Omega_{0}\right) \\
& =\exp \left[-d \mu^{-1} \overline{\mathbf{K}}_{e}(\Theta)\right] \cdot \overline{\mathbf{M}}_{f}\left(\Theta, \Omega_{0}\right) \cdot \exp \left[-d \mu^{-1} \overline{\mathbf{K}}_{e}\left(\Omega_{0}\right)\right] \\
& +\mu^{-1} \int_{-d}^{0} d z^{\prime} \exp \left[z^{\prime} \mu^{-1} \overline{\mathbf{k}}_{e}(\Theta)\right] \cdot \overline{\mathbf{P}}\left(\Theta, \Omega_{0}\right) \cdot \exp \left[z^{\prime} \mu^{-1} \overline{\mathbf{k}}_{e}\left(\Omega_{0}\right)\right] \\
& +\mu^{-1} \int_{-d}^{0} d z^{\prime} \exp \left[z^{\prime} \mu^{-1} \overline{\mathbf{k}}_{e}(\Theta)\right] \cdot \int d \Theta^{\prime} \sin \theta^{\prime} \overline{\mathbf{P}}\left(\Theta, \Theta^{\prime}\right) \\
& \cdot \exp \left[\left(-d-z^{\prime}\right) \mu^{\prime-1} \overline{\mathbf{\kappa}}_{e}\left(\Theta^{\prime}\right)\right] \cdot \overline{\mathbf{M}}_{f}\left(\Theta^{\prime}, \Omega_{0}\right) \cdot \exp \left[-d \mu_{0}^{-1} \overline{\mathbf{\kappa}}_{e}\left(\Omega_{0}\right)\right] \\
& +\mu^{-1} \exp \left[-d \mu^{-1} \overline{\mathbf{K}}_{e}(\Theta)\right] \cdot \int d \Theta^{\prime \prime} \sin \theta^{\prime \prime} \overline{\mathbf{M}}_{f}\left(\Theta, \Omega^{\prime \prime}\right) \\
& \cdot \int_{-d}^{0} d z^{\prime} \exp \left[\left(-d-z^{\prime}\right) \mu^{\prime \prime-1} \overline{\mathbf{\kappa}}_{e}\left(\Omega^{\prime \prime}\right)\right] \cdot \overline{\mathbf{P}}\left(\Omega^{\prime \prime}, \Omega_{0}\right) \cdot \exp \left[z^{\prime} \mu_{0}^{-1} \overline{\mathbf{\kappa}}_{e}\left(\Omega_{0}\right)\right] \\
& +\mu^{-1} \exp \left[-d \mu^{-1} \overline{\mathbf{k}}_{e}(\Theta)\right] \cdot \int d \Theta^{\prime \prime} \sin \theta^{\prime \prime} \overline{\mathbf{M}}_{f}\left(\Theta, \Omega^{\prime \prime}\right) \\
& \cdot \exp \left[\left(-d-z^{\prime}\right) \mu^{\prime \prime-1} \overline{\mathbf{\kappa}}_{e}\left(\Omega^{\prime \prime}\right)\right] \cdot \int d \Theta^{\prime} \sin \theta^{\prime} \overline{\mathbf{P}}\left(\Theta^{\prime \prime}, \Theta^{\prime}\right) \\
& \cdot \exp \left[\left(-d-z^{\prime}\right) \mu^{\prime-1} \overline{\mathbf{K}}_{e}\left(\Theta^{\prime}\right)\right] \cdot \overline{\mathbf{M}}_{f}\left(\Theta^{\prime}, \Omega_{0}\right) \cdot \exp \left[-d \mu_{0}^{-1} \overline{\mathbf{\kappa}}_{e}\left(\Omega_{0}\right)\right]
\end{aligned}
$$

The VRT equation of the symbols are as follows: the Stokes vector I represents the intensity of radiation polarized wave; extinction matrix $\overline{\overline{\mathbf{k}}}_{\mathrm{e}}$ is Stokes vector scattering and absorption coefficient matrix in particle layer; phase transformation matrix $\overline{\mathbf{P}}\left(\Theta_{i}, \Theta_{s}\right)$ is the scattering transmission matrix from $\left(\theta_{i}, \varphi_{i}\right)$ to $\left(\theta_{s}, \varphi_{s}\right), \mu_{0}=\cos \theta_{0}, \mu=\cos \theta$, $\mu^{\prime}=\cos \theta^{\prime} \quad, \quad \mu^{\prime \prime}=\cos \theta^{\prime \prime}$, uplink $\Theta=(\theta, \varphi)$, downlink $\Omega=(\pi-\theta, \varphi)$.

By IEM rough surface's Mueller matrix into $\overline{\mathbf{M}}_{f}$ and extinction matrix with random orientation and phase matrix, then we can obtain the VRT model of sea ice. Without considering the higher order scattering coupling between particles, the particle (air bubbles ) Mueller matrix solution is obtained, according to the scattering volume ratio the total solution of Mueller matrix can be obtained.

To describe the incidence of elliptically polarized wave with the elliptical angle $\chi\left(-45^{\circ} \leq \chi \leq 45^{\circ}\right)$ and the azimuth angle $\psi\left(0^{\circ} \leq \psi \leq 180^{\circ}\right)$, incidence of normalized Stoke vector can be expressed as,
$\overline{\mathbf{I}}_{0}=\left[\begin{array}{llll}\frac{1}{2}(1-\cos 2 \chi \cos 2 \psi) & \frac{1}{2}(1+\cos 2 \chi \cos 2 \psi) & -\cos 2 \chi \sin 2 \psi & \sin 2 \chi\end{array}\right]^{\mathrm{T}}$.

It's easy to see, when $\chi=0^{\circ}, \psi=0^{\circ}$ or $180^{\circ}$, for horizontal polarization $(\mathrm{h}$ ) for vertical polarization ( $\mathrm{v}$ ). The polarization scattering of received power in the backscattering direction is

$$
P_{n}=\frac{1}{2} I_{v s}(I-\cos 2 \chi \cos 2 \psi)+\frac{1}{2}(1+\cos 2 \chi \cos 2 \psi) I_{h s}+\frac{1}{2} U_{s} \cos 2 \chi \sin 2 \psi+\frac{1}{2} V_{s} \sin 2 \chi
$$

where the subscript $\mathrm{n}$ denotes the incident wave is normalized, $\mathbf{I}_{s}=\overline{\mathbf{M}} \cdot \mathbf{I}_{0}$ is the scattering vector Stokes. In this way, the same polarization and polarization backscattering coefficients $\sigma_{c}, \sigma_{d}$ are defined as

$$
\sigma_{c}=4 \pi \cos \theta_{0} P_{n} \quad \sigma_{d}=4 \pi \cos \theta_{0}\left(I_{v s}+I_{h s}-P_{n}\right)
$$

Substitute $\chi=0^{\circ}, \psi=0^{\circ}$ or $\chi=0^{\circ}, \psi=90^{\circ}$, then horizontal polarization backscatter coefficient $\sigma_{h h}$, vertical polarization backscattering coefficient $\sigma_{v v}$ and cross polarization backscattering coefficient $\sigma_{h v}$ can be obtained :

$$
\begin{aligned}
\sigma_{h h} & =4 \pi \cos \theta_{0} \bar{M}(2,2), \sigma_{v v}=4 \pi \cos \theta_{0} \bar{M}(1,1), \\
\sigma_{h v} & =4 \pi \cos \theta_{0} \bar{M}(1,2)
\end{aligned}
$$

$\bar{M}(1,1), \bar{M}(2,2), \bar{M}(1,2)$ are the corresponding elements of the Mueller matrix.

\section{Sea ice model settings}

In the process of ice formation in early stage, when the sea is calm without wind, the longer ice crystals become an ice layer with very smooth surface. If the sea is not calm, crystal is suspended by wind and waves, and then spongy ice is formed by accumulation of ice crystals. As the ice grows, the hardened spongy ice forms a pancake ice with rough surface. Usually in the river estuary near the mouth of the coastal, the sea water is of low salinity. This leads to ice of low salinity, which is quite different in the dielectric constants from sea ice formed in the same period.

Land ice's surface is smooth. The larger the area is, features in the SAR image have lower brightness. Tide caused the sea ice to move vertically and horizontally, the broken of land ice edge is caused by fracture. Due to the increase of surface roughness, scattering is increased, which shows larger region of high brightness in remote sensing images. Fresh water ice and low salinity ice's backscattering are higher, showing in the SAR image with highlight regions, so it cannot be distinguish well between these ice. Compared with SAR, VHF radar has better penetration depth, which can better distinguish the medium under sea ice and have advantages to distinguish different types of sea ice.

By changing electric constant of the physical parameters [7] in sea ice, we simulate radar echo numerically. 
TABLE I Sea Ice Parameters

\begin{tabular}{|c|c|c|c|c|}
\hline \multicolumn{5}{|c|}{ Frequency $=100 \mathrm{MHz}$} \\
\hline & Normal ice & $\begin{array}{c}\text { Low salinity } \\
\text { ice }\end{array}$ & Pan ice & Land ice \\
\hline $\begin{array}{l}\text { Rough surface } \\
\text { between } 01\end{array}$ & $\begin{array}{l}\text { Fluctuation } \\
\text { variance } 2 \mathrm{~cm} \\
\text { Correlation } \\
\text { length } 15 \mathrm{~cm}\end{array}$ & $\begin{array}{l}\text { Fluctuation } \\
\text { variance } \\
2 \mathrm{~cm} \\
\text { Correlation } \\
\text { length } 15 \mathrm{~cm}\end{array}$ & $\begin{array}{l}\text { Fluctuation } \\
\text { variance } \\
3 \mathrm{~cm} \\
\text { Correlation } \\
\text { length } 15 \mathrm{~cm}\end{array}$ & $\begin{array}{l}\text { Fluctuation } \\
\text { variance } \\
2 \mathrm{~cm} \\
\text { Correlation } \\
\text { length } 15 \mathrm{~cm}\end{array}$ \\
\hline $\begin{array}{l}\text { Dielectric } \\
\text { constant of } \\
\text { pure ice and } \\
\text { salt solution } \\
\text { impurity } \varepsilon_{1}\end{array}$ & $\begin{array}{c}3.3280 \\
+\mathrm{i} 0.0125 \\
\text { Salinity } \\
S_{i}=10 \% 0\end{array}$ & $\begin{array}{c}2.8847 \\
+\mathrm{i} 0.0021 \\
\text { Salinity } \\
S_{i}=1 \% \mathrm{o}\end{array}$ & $\begin{array}{l}3.3280 \\
+\mathrm{i} 0.0125 \\
\text { Salinity } \\
S_{i}=10 \%\end{array}$ & $\begin{array}{c}3.3280 \\
+\mathrm{i} 0.0125 \\
\text { Salinity } \\
S_{i}=10 \% 0\end{array}$ \\
\hline $\begin{array}{c}\text { Dielectric } \\
\text { constant of air } \\
\text { bubbles } \varepsilon_{s}\end{array}$ & \multicolumn{4}{|c|}{$\varepsilon_{0}$} \\
\hline $\begin{array}{l}\text { Size of } \\
\text { bubbles } \\
\text { (radius) }\end{array}$ & \multicolumn{4}{|c|}{$0.025 \mathrm{~cm}$} \\
\hline $\begin{array}{c}\text { Orientation of } \\
\text { bubbles }\end{array}$ & \multicolumn{4}{|c|}{ random distribution } \\
\hline $\begin{array}{c}\text { Fraction } \\
\text { volume of air } \\
\text { bubbles }\end{array}$ & \multicolumn{4}{|c|}{0.1} \\
\hline $\begin{array}{l}\text { Dielectric } \\
\text { constant of } \\
\text { layer } 2 \mathcal{E}_{2}\end{array}$ & $\begin{array}{c}\text { Sea water } \\
85.3243 \\
+\mathrm{i} 54.9204\end{array}$ & $\begin{array}{l}\text { Sea water } \\
87.4331 \\
+28.9199 \mathrm{i}\end{array}$ & $\begin{array}{c}\text { Sea water } \\
85.3243 \\
+\mathrm{i} 54.9204\end{array}$ & $\begin{array}{l}\text { Rock } \\
6+\mathrm{i} 0.1\end{array}$ \\
\hline $\begin{array}{c}\text { Rough surface } \\
\text { between } 12\end{array}$ & \multicolumn{3}{|c|}{$\begin{array}{l}\text { Fluctuation variance } \\
\qquad 2 \mathrm{~cm} \\
\text { Correlation length } \\
20 \mathrm{~cm}\end{array}$} & $\begin{array}{l}\text { Fluctuation } \\
\text { variance } 2 \mathrm{~cm} \\
\text { Correlation } \\
\text { length } 40 \mathrm{~cm}\end{array}$ \\
\hline
\end{tabular}

\section{The radar echo simulation}

Radar is settled on aircraft, which sends FM pulse to ground, using the pulse compression techniques for improving range resolution. Through the synthetic aperture radar imaging algorithm, we get the high resolution echo images. In order to detect the sea ice layer's structure, the VHF band has a very high penetration capability, with $100 \mathrm{MHz}$ bandwidth and carrier frequency of $100 \mathrm{MHz}$. By pulse compression, the range resolution can reach $1.5 \mathrm{~m}$.

In this paper, we simulate sea ice with radar echo contour formation in the distance direction. In this paper, the radar pulse is Gauss incident pulse wave [8] with amplitude modulation. Half power width is set to $5 \mathrm{~ns}$, which can achieve $1.5 \mathrm{~m}$ resolution in distance direction.

The model parameters are typical: sea ice thickness is $100 \mathrm{~cm}$ and incidence angle is $30^{\circ}$. Fig. 2 gives four model's (parameters as shown in Table 1 ) radar echo of the polarimetric channels ( VV, $\mathrm{HH}$ ).
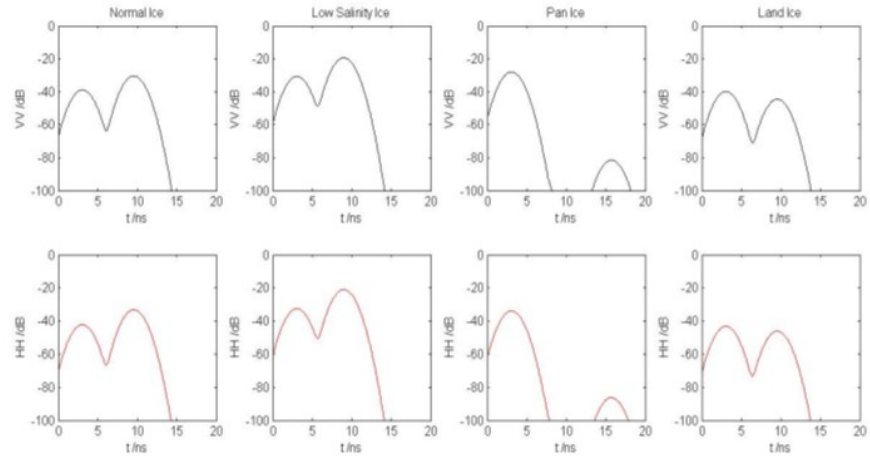

Fig .2 Radar echo of the polarimetric channels ( VV, HH )

Compare four results of radar echo, and the following conclusion is revealed: the decrease of salinity leads to the decreases of imaginary part of dielectric constant and attenuation of sea ice layer decreases, therefore sea water with low salinity leads to increase of dielectric constant's real part and decrease the imaginary part comparing with normal salinity, and results in the increase of bottom echo reflection and absorption reduction. Pan ice's surface is rougher than common ice, and increase of the roughness will significantly enhance the scattering of the surface, so the sea ice scattering decreases, brought by the weakened coherent transmission. The coherent transmission influences the bottom scattering mostly, so the scattering also decreases with the increase of surface roughness. Compared with land ice and sea ice, water makes echoes from the bottom increase significantly.

According to the pulse echo simulation results, it can be seen that VHF radar echo reflects the characteristics and layer structures of sea ice preliminarily. With the application of high resolution VHF radar technology, different radar echo pixel is array to form radar echo images in the horizontal direction. Now assume a terrain structure as follows: one island, above sea level, and sea surface are covered with ice. The cross section is shown in Fig. 3, based on its medium dielectric constant, thickness, we simulate the profile

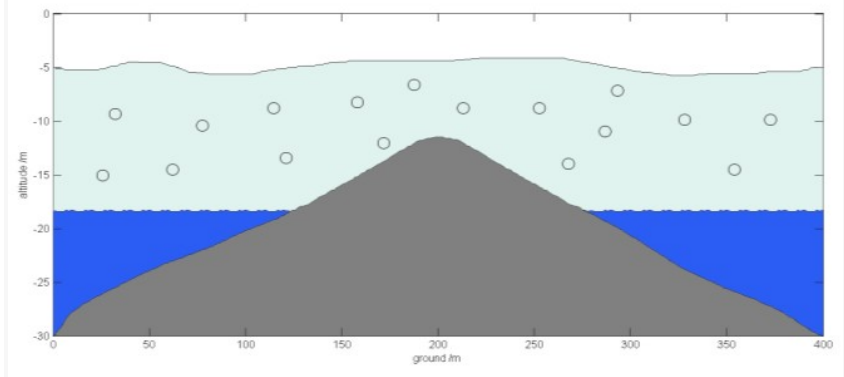

Fig.3 Hypothesis terrain cross section

Calculate echoes in the horizontal direction at intervals of $1.5 \mathrm{~m}, \mathrm{VV}, \mathrm{HH}$ radar echos are shown in Fig. 4. 


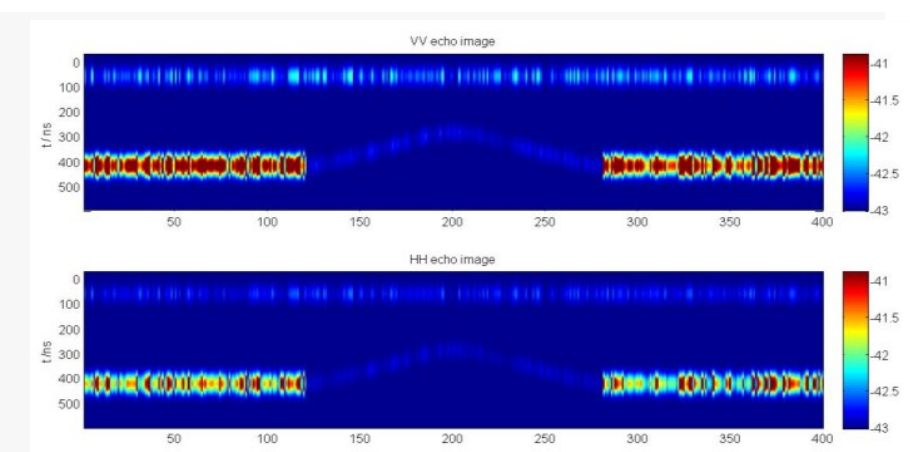

Fig.4 Two-dimensional radar echo map of terrain hypothesis

In Fig. 4 the sea ice surface contour is easily identified, and thickness of ice layer is also easy to identify, which illustrates the feasibility of the proposed model and method. Through the brightness of boundary, we can differentiate land ice and sea ice. By the polarization information of radar echo, ice thickness or landform can be estimated or reversed.

\section{Conclusion}

Based on the time domain Mueller matrix solution, a method for VHF (30-300MHz) radar pulse penetration on sea ice is developed. By changing model parameters such as the layer thickness, salinity, dielectric constants and interfacial roughness, the radar pulse echoes on sea ice are numerically simulated to verify the feasibility of this method. From the simulation results, the radar echo and sea ice types are closely related, and salinity, along with sea-ice roughness significantly change the intensity distribution of radar echo. By the difference in radar echo, we can differentiate different sea ice types.

\section{References}

[1] BAI Shan, LIU Qinzheng, WU huiding. "Relation of ice conditions to climate change in the Bohai Sea of China" [J]. Acta Oceanalogica Sinica, 2001, 20(3): 331-342.

[2] C. Mavrocordatos, E. Attema, and M. Davidson, "Development ofASIRAS (Airborne SAR/Interferometric Altimeter System)," in Proc.IGARSS, 2004, pp. 2465-2467.

[3] Y-Q Jin "Remote sensing of sea ice by multi-frequency micro wave radiometer and numerical modeling of radiative transfer." Remote sensing of environment China, 1992, 7 (1) : 32 40

[4] Feng Xu and Y.-Q. Jin, "Multiparameter inversion of a layer of vegetation canopy over rough surface from the system response function based on the mueller matrix solution of pulse echoes," IEEE

Transactions on Geoscience and Remote Sensing, vol.44, no.7 pp.20032015, July 2006

[5] Y-Q. Jin, "A Mueller Matrix Approach to complete polarimetric scattering from a layer of non-uniformly oriented, non-spherical scatters, ” J. Quant. Spectrosc. Radiat. Transfer, 1992, 48 (3) : 295-306

[6] Ulaby, F.T., Moore R.K. and Fung A.K., "Microwave Remote Sensing, Vol. 3, From Theory to Applications", Artech House, 1986

[7] Klein L. A. and Swift C. T., "An improved model for the dielectric constant of sea water at microwave frequency". IEEE Trans Antennas Propag. 1977, 25:104 111. 32 40.

[8] Y.-Q. Jin and Feng Xu,"Monitoring and early warning the debris flow and landslides using HF radar pulse echoes from layering land media" SCIENCECHINA:Earth Sciences 2012-02: 230-237 